\title{
Joris-Karl Huysmans. De Degas à Grünewald, dir. S. GUÉGAN et A. GUYAUX
}

\section{Ida Merello}

\section{OpenEdition}

\section{Journals}

\section{Edizione digitale}

URL: https://journals.openedition.org/studifrancesi/32113

DOI: 10.4000/studifrancesi.32113

ISSN: 2427-5856

\section{Editore}

Rosenberg \& Sellier

\section{Edizione cartacea}

Data di pubblicazione: 1 août 2020

Paginazione: 437-432

ISSN: 0039-2944

\section{Notizia bibliografica digitale}

Ida Merello, «Joris-Karl Huysmans. De Degas à Grünewald, dir. S. guégan et A. guyaux», Studi Francesi

[Online], 191 (LXIV | II) | 2020, online dal 01 septembre 2020, consultato il 18 septembre 2021. URL: http://journals.openedition.org/studifrancesi/32113; DOI: https://doi.org/10.4000/studifrancesi. 32113

Questo documento è stato generato automaticamente il 18 septembre 2021.

\section{(c) (i) (9) $\Theta$}

Studi Francesi è distribuita con Licenza Creative Commons Attribuzione - Non commerciale - Non opere derivate 4.0 Internazionale. 


\title{
Joris-Karl Huysmans. De Degas à Grünewald, dir. S. GUÉGAN et A. GUYAUX
}

\author{
Ida Merello
}

\section{NOTIZIA}

Joris-Karl Huysmans. De Degas à Grünewald, dir. S. GUÉGAN et A. GUYAUX, Musée d'Orsay Gallimard, 2019, $224 \mathrm{pp}$.

1 Il volume ha per occasione la mostra presso il museo di Strasburgo, dedicata a Joris-Karl Huysmans, De Degas a Grünewald sous le regard de Francesco Vezzoli (artista che ha inserito nell'esposizione alcune proprie opere di omaggio allo scrittore). Oltre che costituire un catalogo delle opere, il volume offre una lettura approfondita dei giudizi critici di Huysmans attraverso gli specialisti del settore.

2 Stéphane GUÉGAN (Toute vérité est bonne à dire, pp. 27-31) segnala l'evoluzione del gusto di Huysmans facendo notare che lo scrittore non pone come inizio della sua raccolta di critica l'articolo sugli impressionisti del 1876, bensì quello apparso sul "Voltaire" nel 1879, dove Degas è l'artista più apprezzato e il sostegno al Salon des Indépendants è incondizionato. In quell'occasione Raffaëlli è individuato come l'illustratore ideale di $A$ Rebours; mentre Forain sarà scelto per il frontespizio della seconda edizione di Marthe.

3 Francesca GUGLIELMI (Les débuts de Huysmans dans la critique d'art, pp. 54-67) ricorda come i tentativi di pubblicare Le drageoir aux épices abbiano favorito i canali per pubblicare la critica d'arte. Lo scrittore apprezza non tanto i paesaggi impressionisti, quanto invece Degas, che gli sembra più realista. Mentre i suoi rapporti con "La République des lettres" e con Mendès si incrinano presto, Huysmans grazie alle conoscenze ottenute moltiplica l'attività su riviste francesi e successivamente belghe. 
4 Aude JEANNEROT (Huysmans et l'art officiel: un critique d'opposition, pp. 68-79) legge i giudizi artistici di Huysmans anche alla luce delle convinzioni religiose e politiche dello scrittore. Mentre tende alla protezione degli emarginati, e quindi è sempre dalla parte del Salon des Indépendants, Huysmans cambia parere sugli artisti nel momento in cui si affermano di più. È questo per esempio il caso di Manet, di cui inoltre non apprezza i quadri a ispirazione repubblicana. Non sopporta neppure $i$ quadri a ispirazione militarista e patriottica che furoreggiavano dopo la sconfitta di Sédan.

André GUYAuX (Le temps de Degas, Redon, Moreau e Rops, pp. 92-113) ricorda che al momento della stesura di Marthe, $\mathrm{H}$. è molto legato agli impressionisti, perché ancora vicino al gruppo di Médan, e cerca in pittura tutto quello che gli sembra un'espressione di verità. La recensione di Nana di Manet rivela ancora il gusto parigino della vita dei caffè e delle mondane. Guyaux avverte un incrocio, quasi un'ekphrasis, tra la critica di Huysmans, Nana e Marthe. Sono le figure delle demi-mondaines a interessarlo, come espressione della pariginità. Ancora l'anno successivo a colpirlo di più, in un quadro di Gervex, Rolla, è la figura femminile con caratteristiche simili. A partire dal 1879, stabilisce un legame tra Degas e il naturalismo: Degas diventa il suo pittore della vita moderna, e ne esalta la modernità tra il 1876 e il 1882, accompagnandola con l'ostilità nei confronti delle manifestazioni ufficiali dell'arte: è il Salon des Indépendants quello che predilige. Nel frattempo ha incontrato Forain, che illustrerà la seconda edizione di Marthe; mentre conosce Raffaëlli e Redon nel momento in cui sta passando dal naturalismo ad A rebours. Redon lo affascina, sia per la personalità che per lo stile. L'A. fa ben vedere che Redon ha tutto per piacere a Huysmans: vive in disparte, non cerca la gloria, è autonomo dalle scuole. Huysmans lo vanta con i suoi amici, fa comprare le sue opere, lo presenta a Mallarmé, ma soprattutto, mentre offre suggerimenti sull'uso del colore, impara da Redon una nuova spiritualità. Con Moreau invece non si è creato lo stesso rapporto, forse perché Moreau è troppo intellettuale e inattuale. L'A. riporta lo scambio tra Moreau e Pissarro al momento dell'uscita dell'Art Moderne: Pissarro (a sua volta poco messo in valore) lamenta l'assenza di Cézanne e la scarsa stima per Monet. Nelle giustificazioni e parziali ammissioni di colpa, Huysmans ribadisce la sua ricerca di individualità libere. Ma i nuovi pittori che individua non hanno la forza di un Moreau o di un Redon. Nel suo volume successivo, Certains, Moreau e Redon non compaiono perché li salvaguarda per À rebours. Il suo sguardo sull'arte moderna sta cambiando: non c'è più una fiducia nelle dottrine collettive, ma nella spigolatura personale di soggetti amati. Riprende pagine già pubblicate sui giornali a proposito di Whistler, $\mathrm{e}$ aggiunge un capitolo su Rops, che ha appena illustrato le Diaboliques e le Sataniques di Barbey. Ma la conclusione del volume giù annuncia la conversione.

Mireille DOTTIN-ORSINI e Daniel GRojNowsKi (Huysmans, Moreau et Salomé, pp. 114-123) approfondiscono la questione delle relazioni tra Moreau e Huysmans, mostrando come l'interesse per Moreau sia in contro tendenza nel momento naturalista dello scrittore. Salomé lo affascina e Des Esseintes possiede quegli originali che Huysmans non può permettersi. L'attrazione erotica si rifrange in un erotismo che investe non solo la danzatrice, ma anche il suo corpo suppliziato. Tuttavia la figura preferenziale sarà quella della giovane mendicante nel quadro su re Cophetua di Burne-Jones.

7 Pierre VAISSE (L'Art religieux en République, pp. 140-157) ricorda la netta demarcazione, sotto la terza Repubblica, tra progresso tecnico e ideali repubblicani da un lato e spiritualismo simbolista dall'altro, senza però che quest'ultimo coincida con il cattolicesimo. Nello stesso tempo le grandi opere a carattere religioso sono ordinate 
dallo Stato e improntate all'enfasi, o sono dettate dall'estetismo. Huysmans sa invece cogliere la grandezza dei primitivi fiamminghi, lasciando il segno più di Verhaeren o Wyzewa, per la sua fama di scrittore in Francia.

8 André GUYAUX "Le seul art véridique est grand": Huysmans et les primitifs, pp. 158-168, sottolinea che Huysmans non abiura la lotta per i pittori della vita moderna, ma all'inizio degli anni Ottanta si rende conto del loro materialismo, per cui si volge ai pittori dell'immaginario (Redon, Moreau, Rops) attribuendo loro un'ascendenza nei pittori Primitivi. Non già italiani, sospetti di malcelata sodomia, ad eccezione del Beato Angelico, bensì fiamminghi, visti per di più in loco, insieme all'amico Arij Prins. Per l'Italia fa solo un'altra eccezione, per il pittore rinascimentale Bartolomeo Veneto, che, malgrado il desiderio di isolare i Primitivi dal Rinascimento, accosta non solo per l'analogia del trittico, ma anche per l'oscillazione costante in lui tra lussuria e purezza.

9 Estelle PIETRZYK (Huysmans ou l'art de l'inventaire, pp. 168-179) mostra il gusto del catalogo nelle opere di Huysmans, dal Drageoir aux épices ad À rebours, dagli oggetti insignificanti ai quadri ai soprammobili rari. Oggetti che si traducono in uno stile "artiste", che rivela il gusto del parossismo e uno sguardo mobile, rappresentato con tecniche già cinematografiche, in avanti rispetto alla teoria. 